\title{
Wavelet analysis of Jones-matrix images corresponding to polycrystalline networks of biological crystals in diagnostics of tuberculosis
}

\author{
N.I. Zabolotna ${ }^{1}$, V.O. Balanetska ${ }^{2}$, O.Yu. Telenga ${ }^{3}$, V.O. Ushenko ${ }^{3}$ \\ ${ }^{1}$ Vinnytsia National Technical University, Department for Laser and Optoelectronic Technique, \\ 95, Khmelnytske shose, 21021 Vinnytsia, Ukraine. \\ ${ }^{2}$ Bukovina State Medical University, Department of Biophysics and \\ Medical Informatics, 2, Teatralnaya Sq., 58012 Chernivtsi, Ukraine. \\ ${ }^{3}$ Chernivtsi National University, Department for Optics and Spectroscopy, \\ 2, Kotsyubinsky str., 58012 Chernivtsi, Ukraine.
}

\begin{abstract}
Determined in this work are analytical interrelations between orientations of optical axes and birefringence of biological crystals as well as characteristic values of elements in the Jones matrix for flat layers of polycrystalline networks, which determine conditions for formation of polarization singularities in laser images. Performed is a complex statistical, correlation and fractal analysis of distributions for the amount of characteristic values inherent to the Jones matrix elements describing layers of saliva taken from a healthy patient and that sick with tuberculosis. The authors have ascertained objective criteria to differentiate optical properties of polycrystalline networks of human saliva in various physiological states. Offered is Jones-matrix diagnostics of tuberculosis.
\end{abstract}

Keywords: laser, polarization, birefringence, Jones matrix, statistical moments, autocorrelation, power spectrum, tuberculosis.

Manuscript received 04.07.11; accepted for publication 16.03.11; published online 30.06.11.

\section{Introduction}

Among the methods of optical diagnostics aimed at biological tissues (BT), the methods of laser polarimetry of optically anisotropic structures in human tissues have been already widely spread [1 - 31]. The main "information product" of using these methods is data for coordinate distributions inherent to Mueller and Jones matrixes typical for BT [1 - 5]. Then, these data can be processed with statistical (statistical moments of the first to fourth orders $[5,6,10,14,19,25,26,30])$, correlation (auto- and mutual-correlation functions [12, 17, 18, 21, 26]), fractal (fractal dimensionalities [5, 6, 25]), singular (distributions of amounts of linear and circularly polarized states), wavelet (sets of wavelet coefficients for various scales of biological crystals [22, 28]) analyses. As a result, one can determine interrelations between a set of these parameters and distributions of optical axis directions as well as the birefringence value inherent to networks of optically single-axis protein (myosin, collagen, elastin, etc.) fibrils in optically anisotropic components of BT layer. Being based on it, developed was a set of methods for diagnostics and differentiation of pathological changes in BT structure, which are related with its degenerative-dystrophic and oncological changes [4 - 6, 12, 19, 20-22, 27, 29, 31].
Progress in the above methods for studying the matrix images of BT was reached in [5]. There, the offered new approach is based on the analysis of coordinate distributions for the so-called "characteristic values" that describe conditions for formation of polarization singularities. Related to these singularities are linear (Lpoints) and circularly (C-points) polarized states. In the case of L-points, the direction of electric field vector rotation is indefinite (singular). For C-points, indefinite is the azimuth of polarization for the electric field vector. Demonstrated in [...] was the efficiency of this approach for Mueller-matrix diagnostics of pathological states in human BT. At the same time, there is a widely spread group of optically anisotropic biological objects, for which the matrix methods of laser polarimetric diagnostics did not yet acquire any wide application. These are optically thin (extinction coefficient $\tau \leq 0.1$ ) layers of various biological liquids (bile, urine, liquor, joint fluid, blood plasma, saliva, etc.). These objects are considerably more accessible for direct laboratory analysis as compared with traumatic methods of BT biopsy. Being based on these reasons, it seems topical to adapt the methods of laser polarimetric diagnostics of optically anisotropic structures observed in BT polycrystalline networks for medical purposes. 
Bearing in mind diagnostics of tuberculosis, our work is aimed at searching possibilities to diagnose and differentiate optical properties of liquid-crystal networks in human saliva by using determination of coordinate distributions for Jones-matrix elements with the following wavelet analysis of distributions inherent to their characteristic (singular) values.

\section{Main analytical interrelations}

As a base for modeling the optical properties of liquidcrystalline networks in human saliva, we took the following conceptions [1-4, 7, 9, 14, 16, 23-27, 30]:

- $\quad$ separate (partial) liquid crystals (...) are optically single-axis and birefringent;

- optical properties of a partial crystal can be exhaustively described with the Jones operator [5]

$$
\begin{aligned}
& \{J\}=\left\|\begin{array}{ll}
J_{11} & J_{12} \\
J_{21} & J_{22}
\end{array}\right\|= \\
& \left\|\begin{array}{cc}
\cos ^{2} \rho+\sin ^{2} \rho \exp (-i \delta) ; & \cos \rho \sin \rho[1-\exp (-i \delta)] ; \\
\cos \rho \sin \rho[1-\exp (-i \delta)] ; & \sin ^{2} \rho+\cos ^{2} \rho \exp (-i \delta) ;
\end{array}\right\| .
\end{aligned}
$$

Here, $\rho$ is a direction of the optical axis; $\delta=2 \pi / \lambda^{\Delta n d}-$ phase shift between orthogonal components $E_{x}$ and $E_{y}$ of the amplitude of illuminating laser wave with the wavelength $\lambda ; \Delta n$ birefringence index of the crystal with the geometric size $d$.

Let us consider the possibility to apply the singular approach in the Jones-matrix images. From the mathematical viewpoint, a singular value of a matrix element $J_{i k}$ complex value is defined by the following conditions:

$$
\left\{\begin{array}{l}
\left(\operatorname{Re} J_{i k}\right)^{2}+\left(\operatorname{Im} J_{i k}\right)^{2}=0 ; \\
\operatorname{Re} J_{i k}=0 ; \\
\operatorname{Im} J_{i k}=0 .
\end{array}\right.
$$

With account of (2), the analytical expressions (1) are transformed to interrelations

$$
\begin{aligned}
& J_{11} \Leftrightarrow\left\{\begin{array}{l}
\sin ^{2} \rho \sin \delta=0 ; \\
\cos ^{2} \rho+\sin ^{2} \rho \cos \delta=0 .
\end{array}\right. \\
& J_{22} \Leftrightarrow\left\{\begin{array}{l}
\cos ^{2} \rho \sin \delta=0 ; \\
\sin ^{2} \rho+\cos ^{2} \rho \cos \delta=0 .
\end{array}\right. \\
& J_{12}=J_{21} \Leftrightarrow\left\{\begin{array}{l}
2 \cos ^{2} \rho \sin ^{2} \rho(1+\cos \delta)=0 ; \\
\sin 2 \rho(1+\cos \delta)=0 .
\end{array}\right.
\end{aligned}
$$

As it follows from (3) to (5), singularities of complex matrix elements $J_{i k}^{*}$ are conditioned by certain (characteristic) values of orientation $\rho^{*}$ and phase $\delta^{*}$ parameters corresponding to liquid-crystalline network

$$
\left\{\begin{array}{l}
\rho^{*}=0^{0} ; \quad \pm 45^{0} ; \quad 90^{0} ; \\
\delta^{*}=0^{0} ; \quad \pm 90^{0} ; 180^{0} .
\end{array}\right.
$$

On the other hand, the relations (6) determine the conditions for formation polarization singular states $L-$ ( $\left.\delta=0^{0} ; 180^{0}\right)$ and $C-\left(\delta= \pm 90^{0}\right)$ of the laser beam by optically single-axis birefringent crystal. Being based on it, one can find the characteristic values of the Jones matrix elements $J_{i k}^{*}$ that define $L$ - and $C$ - states of polarization in a laser image of polycrystalline network:

- $J_{11}=J_{22}=0$ values define $L-$ states of polarization;

- $J_{12}=J_{21}=0$ values define $C$-states of polarization.

It is noteworthy that the analytical consideration of (1) to (6) is related to a partial optically single-axis birefringent crystal. Formed in real biological layers are complex networks of these crystals with different scales of geometric sizes. Therefore, application of the singular analysis to the Jones matrix corresponding to this network requires determining the coordinate distributions of characteristic values $J_{i k}^{*}(x, y)$ in the plane of biological liquid layer. These distributions can be determined by scanning two-dimensional arrays of $J_{i k}$ elements in horizontal direction $x \equiv 1, \ldots, m$ with the step $\Delta x=1 p i x$. Within the limits of every local sampling $\left(1_{\text {pix }} \times n_{\text {pix }}\right)^{(k=1,2, \ldots, \mathrm{m})}$, one has to calculate $N$ characteristic values $J_{i k}(k)=0,-\left(N_{i k}^{(k)}\right)$. In this manner, one finds dependences $N_{i k}(x) \equiv\left(N_{i k}{ }^{(1)}, \quad N_{i k}{ }^{(2)}, \ldots, N_{i k}{ }^{(m)}\right)$ of the amount of characteristic values for matrix elements. On the other hand, these multi-scale dependences can be efficiently analyzed using the wavelet analysis [...]. If as a wavelet function one takes the dependence that possesses a finite base both in coordinate and frequency spaces, then using the scaling and shift of this function-prototype the coordinate distribution of Jones matrix elements can be expanded into the following series

$N_{i k}(x)=\sum_{a, b=-\infty}^{\infty} C_{a b} \Psi_{a b}(x)$,

where $\Psi_{a b}(x)=\Psi(a x-b)$ is the base function that is formed from the function-prototype using the shift $\boldsymbol{b}$ and scaling $\boldsymbol{a}$, while the coefficients of this expansion are defined in the following manner

$C_{a b}=\int \aleph_{i k}(x) \Psi_{a b}(x) d x$

In our work, using the analogy with the investigation [...], as a wavelet function we chose the second derivative of the Gauss function (MHAT wavelet) that possesses a narrow energy spectrum and 
two moments equal to zero (zero and first). The analytical dependence of the MHAT wavelet has the following look:

$\Psi(z)=\frac{d^{2}}{d z^{2}} e^{-z^{2} / 2}=\left(1-z^{2}\right) e^{-z^{2} / 2}$.

The result of wavelet transformation (9) is twodimensional array of amplitudes or the spectrum $W(a, b)$ that in the space "spatial scale a - spatial localization b" provides information on relative contribution of birefringent network in crystals of different scales to the dependence $N_{i k}(x)$. Thus, it opens the possibility to realize a scale-selective analysis of real polycrystalline networks in various biological layers.

\section{Optical setup for making Jones-matrix maps of optically anisotropic biological liquids}

Shown in Fig. 1 is the optical scheme of a polarimeter for measuring the coordinate distributions of elements in the Jones matrix corresponding to biological layers. Illumination of bile samples was carried out using a parallel beam $\left(\varnothing=10^{4} \mu \mathrm{m}\right)$ of the He-Ne laser $(\lambda=$ $0.6328 \mu \mathrm{m}, \mathrm{W}=5.0 \mathrm{~mW}$ ). The polarization illuminator consists of the quarter-wave plates 3,5 and polarizer 4 , which provides formation of the laser beam with an arbitrary polarization state. Using the micro-objective 7 (magnification $4 \mathrm{x}$ ), images of bile layers were projected onto the plane of light-sensitive area (800x600 pixels) in the CCD-camera 10 that provides measurements of structural elements from 2 to $2000 \mu \mathrm{m}$.

The analysis of laser images was performed using the polarizer 9 and quarter-wave plate 8 .

\section{Criteria for estimating the spectra of wavelet coefficients corresponding to the amount of characteristic values inherent to Jones-matrix images describing the layers of human saliva}

Distributions $W(a, b)$ of the amount of characteristic values for elements of the Jones matrix $J_{i k}(m \times n)$ are characterized with the set of statistical moments of the 1st to 4-th orders $M ; \sigma ; A ; \quad E$ calculated using the following relations $[5,6, \quad 25, \quad 30]$ :

$$
\begin{aligned}
& M=\frac{1}{D} \sum_{j=1}^{D}\left|(W(a, b))_{j}\right|, \quad \sigma=\sqrt{\frac{1}{D} \sum_{j=1}^{D}(W(a, b))_{j}{ }^{2}}, \\
& A=\frac{1}{\sigma^{3}} \frac{1}{D} \sum_{j=1}^{D}(W(a, b))_{j}{ }^{3}, \quad E=\frac{1}{\sigma^{4}} \frac{1}{D} \sum_{j=1}^{D}(W(a, b))_{j}{ }^{4} .
\end{aligned}
$$

where $D$ is the number of pixels for which the dependence of characteristic values $N_{i k}$ within the limits of coordinate distribution for Jones-matrix images of elements $J_{i k}$ is determined.

Our analysis of the spectra for wavelet coefficients $W(a, b)$ in distributions $N_{i k}(x)$ was based on the autocorrelation method with using the following function $[12,21,26]$ :

$G(\Delta b)=\frac{1}{m} \int_{1}^{m}\left[W_{a}(b=1 \div x)\right]\left[W_{a}(b-\Delta b)\right] d b$.

Here, $\Delta b$ is the "step" of changing the coordinate $x \equiv b=1 \div m$.

As to parameters characterizing the dependences $G(\Delta b)$, we chose the set of correlation moments from the 1-st to 4-th orders $K_{l=1 ; 2 ; 3 ; 4}$ that are determined similarly to relations (10).

Estimating the degree of self-similarity and reproducibility for different geometric ( $d$ ) scales of the structure inherent to wavelet coefficients $W(a, b)$ of the distributions $N_{i k}(x)$ corresponding to characteristic values of the Jones-matrix elements $J_{i k}(m \times n)$ describing the polycrystalline networks was performed via calculations of the logarithmic dependences for power spectra $\log J[W(a, b)]-\log \left(d^{-1}\right)$. These dependences are approximated using the least-squares method to curves $\Phi(\eta)$. Straight-line parts of these curves enable us to determine the slope angles $\eta_{i}$ and calculate the values of fractal dimensionality for $W(a, b)$ distributions with account of the relations [5, 6, 11, 25]:

$D_{i}(g)=3-\operatorname{tg} \eta_{i}$.

Classification of the distributions $W(a, b)$ for dependences of the amount of characteristic values

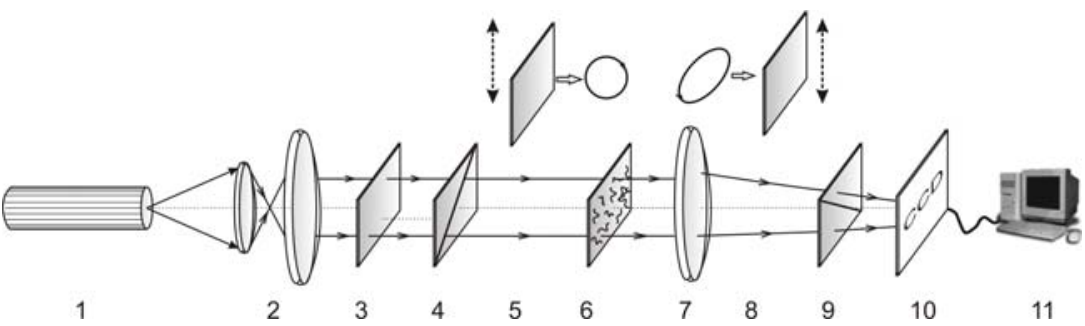

Fig. 1. Optical scheme of the polarimeter: 1 - He-Ne laser; 2 - collimator; 3 - stationary quarter-wave plate; 5 , 8 mechanically movable quarter-wave plates; 4, 9 - polarizer and analyzer, respectively; 6 - studied object; 7 - micro-objective; 10 - CCD camera; 11 - personal computer. 
typical for the matrix elements $J_{i k}(m \times n)$ is carried out in accord with criteria offered in [5]. If the value of the slope angle $\eta=$ const , the dependences $\Phi(\eta)$ for 2 or 3 decades of changing the sizes $d$, than the distributions $W(a, b)$ are fractal. Under condition that several constant slope angles are available in the curve $\Phi(\eta)$, the sets $W(a, b)$ are multi-fractal. When no stable slope angles are available over the whole interval of changing the sizes $d$, the sets $W(a, b)$ are considered as random.

To make this comparative analysis of $\log J[W(a, b)]-\log \left(d^{-1}\right)$ dependences more objective, let us use the conception of spectral moments from the 1-st to 4-th orders $S_{j=1 ; 2 ; 3 ; 4}$ - the relation (7).

\section{Analysis and discussion of experimental results}

As objects of investigation, we used saliva smears of healthy (18 samples) and sick with tuberculosis (17 samples) patients. Fig. 2 shows laser images of opticallyanisotropic structures typical for the samples of both types. The images were obtained for crossed transmission planes of the polarizer 4 and analyzer 9 in the laser polarimeter (Fig. 1).

It can be seen from a comparative analysis of laser images corresponding to liquid-crystalline networks of the studied samples that the level of transmission (birefringence) grows in the case of small-scale $(d=10 \ldots 30 \mu \mathrm{m})$ liquid crystals in saliva of the patients sick with tuberculosis (Fig. 2b). This fact was used as a basis for our wavelet analysis of the dependences $N_{12 ; 21}(x)$ for distributions of characteristic values inherent to $J_{12 ; 21}(m \times n)$ elements in Jones-matrix images. Our choice of just these elements is related with the fact that $J_{12 ; 21}=0$ values characterize the probability of $C-$ point formation, which is related with growth of birefringence in liquid crystals at certain scales of geometric sizes.

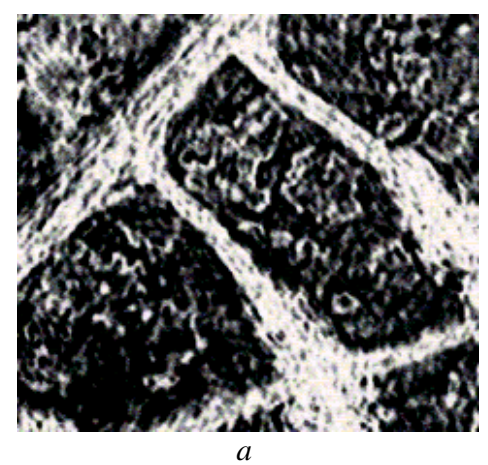

Depicted in Figs 3 and 4 are the results of determining the dependence $N_{12 ; 21}(x)$ (fragment (a)) and the respective spectrum of wavelet coefficients $W_{a, b}(N(x))$ (fragment (b)).

It is seen from the data obtained that the twodimensional array $W_{a, b}(N(x))$ is a complex coordinateinhomogeneous and scale-dependent set of values for wavelet coefficients. Starting from this analysis, it is necessary to use a complex statistical, correlation and fractal analysis of these wavelet spectra for distributions of characteristic values in the Jones-matrix images of liquid-crystalline networks.

Shown in Figs 5 and 6 is the set of coordinate $W_{a=c o n s t, b=1 \div m}\left(N\left(J_{12 ; 21}=0\right)\right), \quad$ autocorrelation $G\left(W_{a=\text { const }, b=1 \div m}\left(N\left(J_{12 ; 21}=0\right)\right)\right) \quad$ and spectral $\log F\left(W_{a, b}\right)-\log d^{-1}$ dependences that characterize the spectra of wavelet coefficients $W_{a, b}(N(x))$ for two scales $a=14$ and $a=70$ of the wavelet function $\Psi_{a, b}$ (relation (9)) for the distributions $N\left(J_{12 ; 21}=0\right)$ corresponding to liquidcrystalline networks in saliva of healthy patient (Figs 3 and 5) and that sick with tuberculosis (Figs 4 and 6).

It is seen from the data obtained that coordinate, autocorrelation and spectral dependences that characterize the sets of wavelet coefficients $W_{a, b}\left(N\left(J_{12 ; 21}=0\right)\right)$ corresponding to distributions of the amount of characteristic values $J_{12 ; 21}=0$ in the Jonesmatrix image $J_{12 ; 21}(m \times n)$ of liquid-crystal network in the layer of saliva taken from a healthy patient and from that sick with tuberculosis are individual in every scale of a MHAT wavelet.

From the quantitative viewpoint, the differences between coordinate $W_{a=\text { const }, b=1 \div m}\left(N\left(J_{12 ; 21}=0\right)\right)$, autocorrelation $G\left(W_{a=\text { const }, b=1 \div m}\left(N\left(J_{12 ; 21}=0\right)\right)\right)$ and spectral $\log F\left(W_{a, b}\right)-\log d^{-1}$ dependences characterize

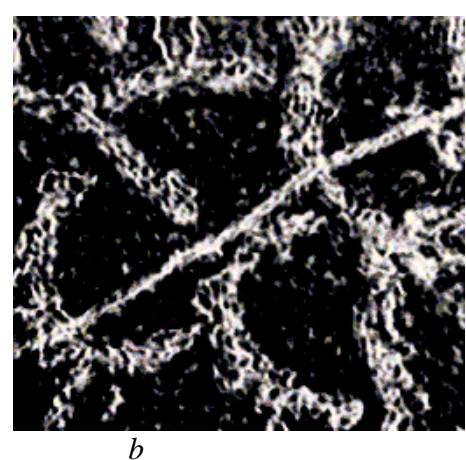

Fig. 2. Polarization images of dendrite polycrystalline networks typical for human saliva in different physiological states. See explanations in the text. 

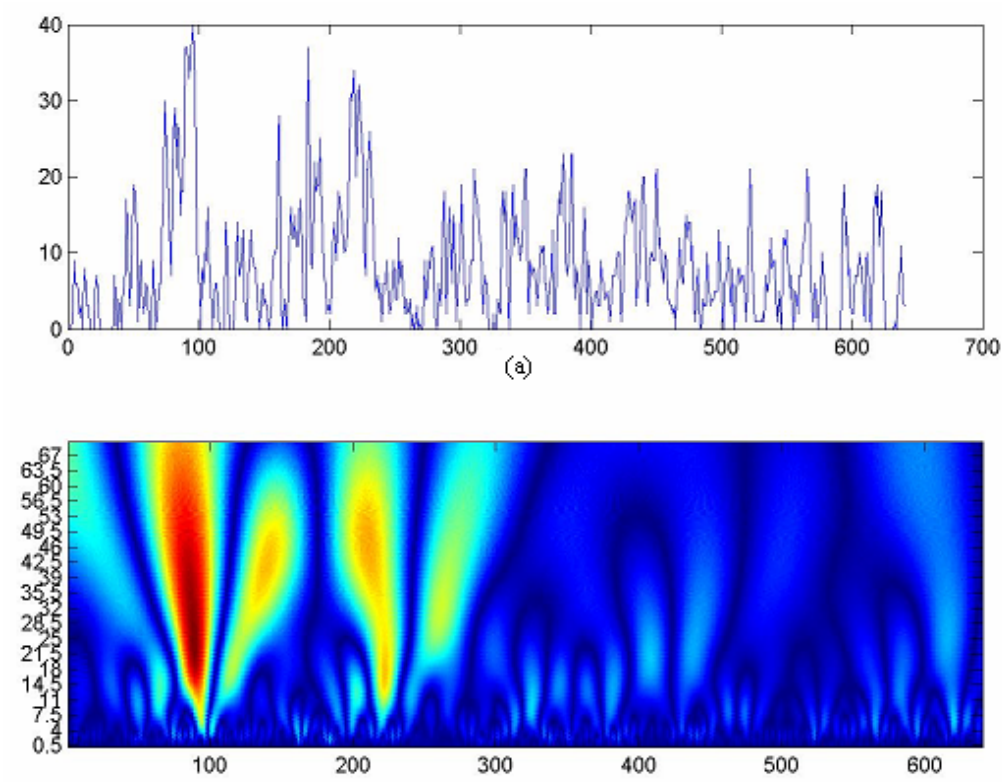

(6)

Fig. 3. Spectrum of wavelet coefficients $W_{a, b}(N(x))$ for the distribution $N_{12 ; 21}(x)$ in the Jones-matrix image $J_{12 ; 21}(m \times n)$ corresponding to liquid-crystalline network of a saliva layer taken from a healthy patient.

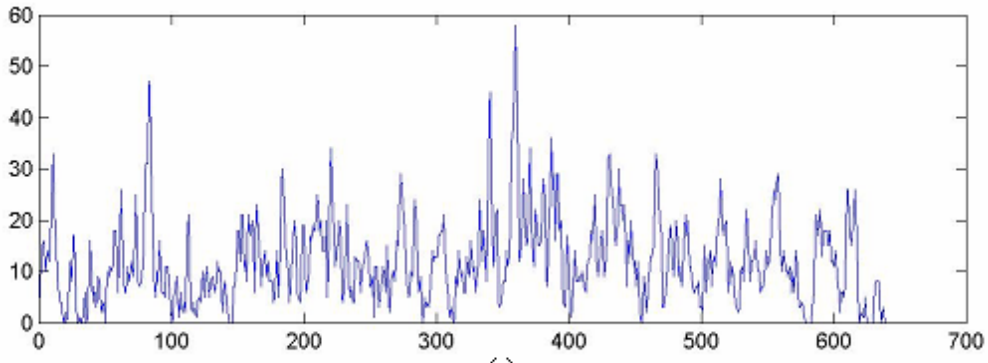

(a)

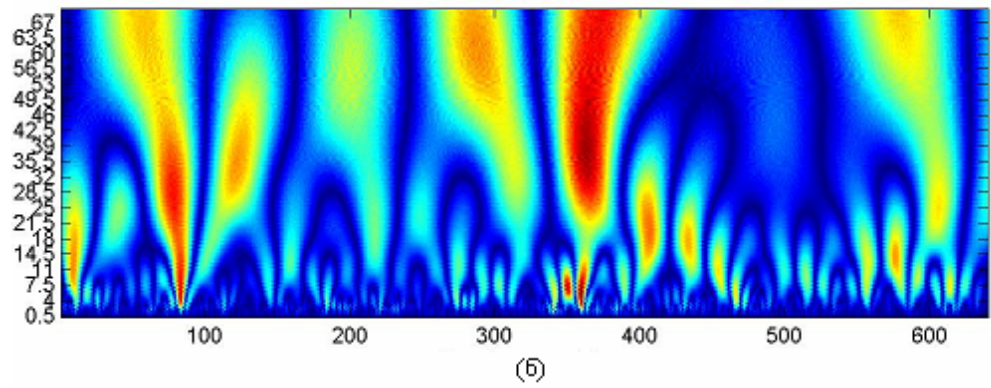

Fig. 4. Spectrum of wavelet coefficients $W_{a, b}(N(x))$ for the distribution $N_{12 ; 21}(x)$ in the Jones-matrix image $J_{12 ; 21}(m \times n)$ corresponding to liquid-crystalline network of a saliva layer taken from a patient sick with tuberculosis.

the values and ranges for changing the set of statistical, correlation and spectral moments of the 1-st to 4-th orders (Table 1).

It is seen from the analysis of the data summarized in Table 1 that changes in values of statistical moments of the 1-st to 4-th orders, which characterize the distributions of wavelet coefficients $W_{a, b}\left(N\left(\aleph_{12 ; 21}=0\right)\right)$ possess extreme values for the scale $a=14$ of the MHAT - function.
We have ascertained the following tendencies of changes in the statistical moments of the 1-st to 4-th orders:

- $\quad$ mean value $M\left(W_{a=14}\left(N\left(J_{12 ; 21}=0\right)\right)\right)$ increases by 1.9 times;

- $\quad$ dispersion $\sigma\left(W_{a=14}\left(N\left(J_{12 ; 21}=0\right)\right)\right)$ increases by 2.85 times;

- $\quad$ skewness $A\left(W_{a=14}\left(N\left(J_{12 ; 21}=0\right)\right)\right)$ increases by 3.2 times; 


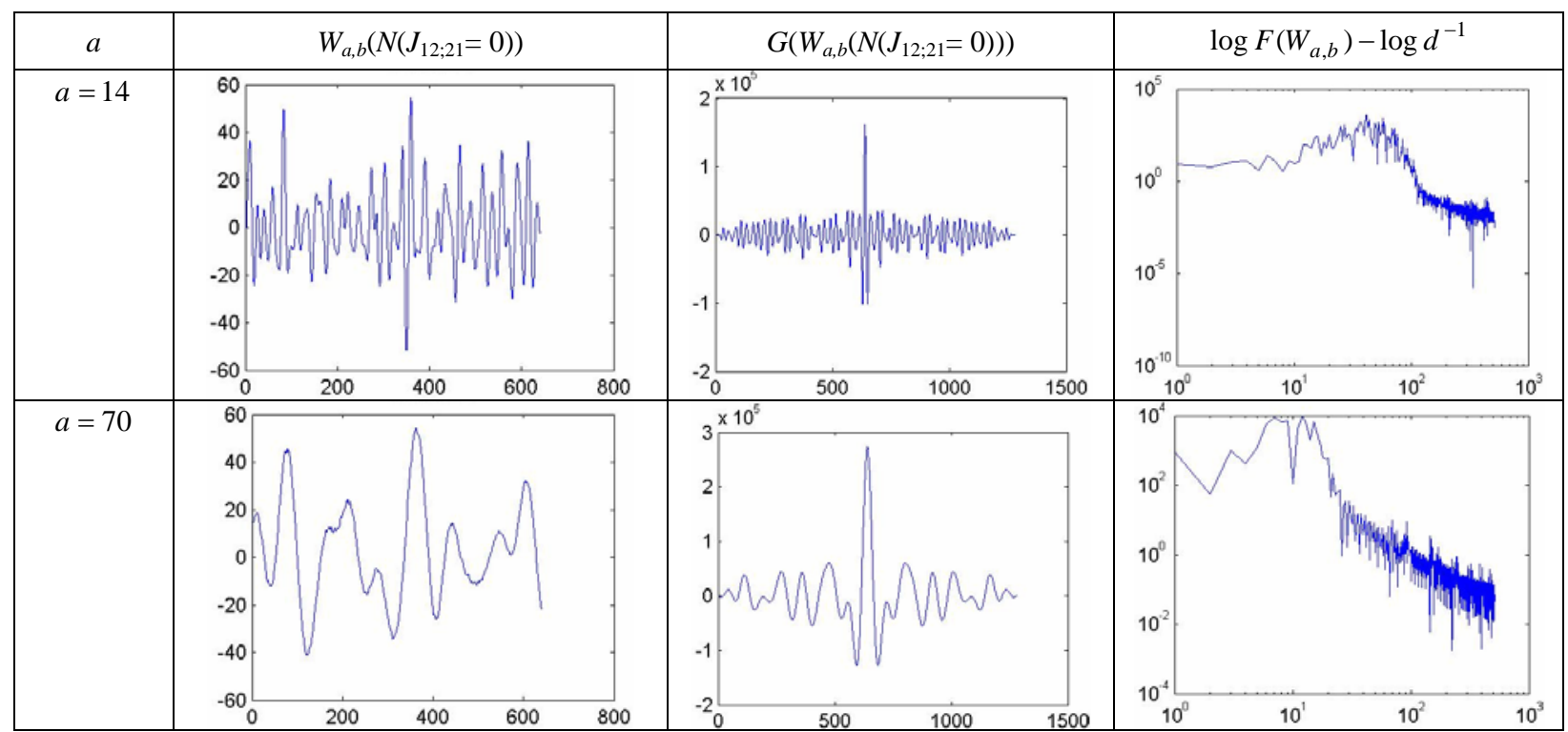

Fig. 5. Coordinate (left column), autocorrelation (central column) and spectral (right column) dependences that characterize the spectrum of wavelet coefficients $W_{a, b}\left(N\left(J_{12 ; 21}=0\right)\right)$ for the distribution of characteristic values in the Jones-matrix image $J_{12 ; 21}(m \times n)$ of liquid-crystal network in the layer of saliva taken from a healthy patient. See explanations in the text.

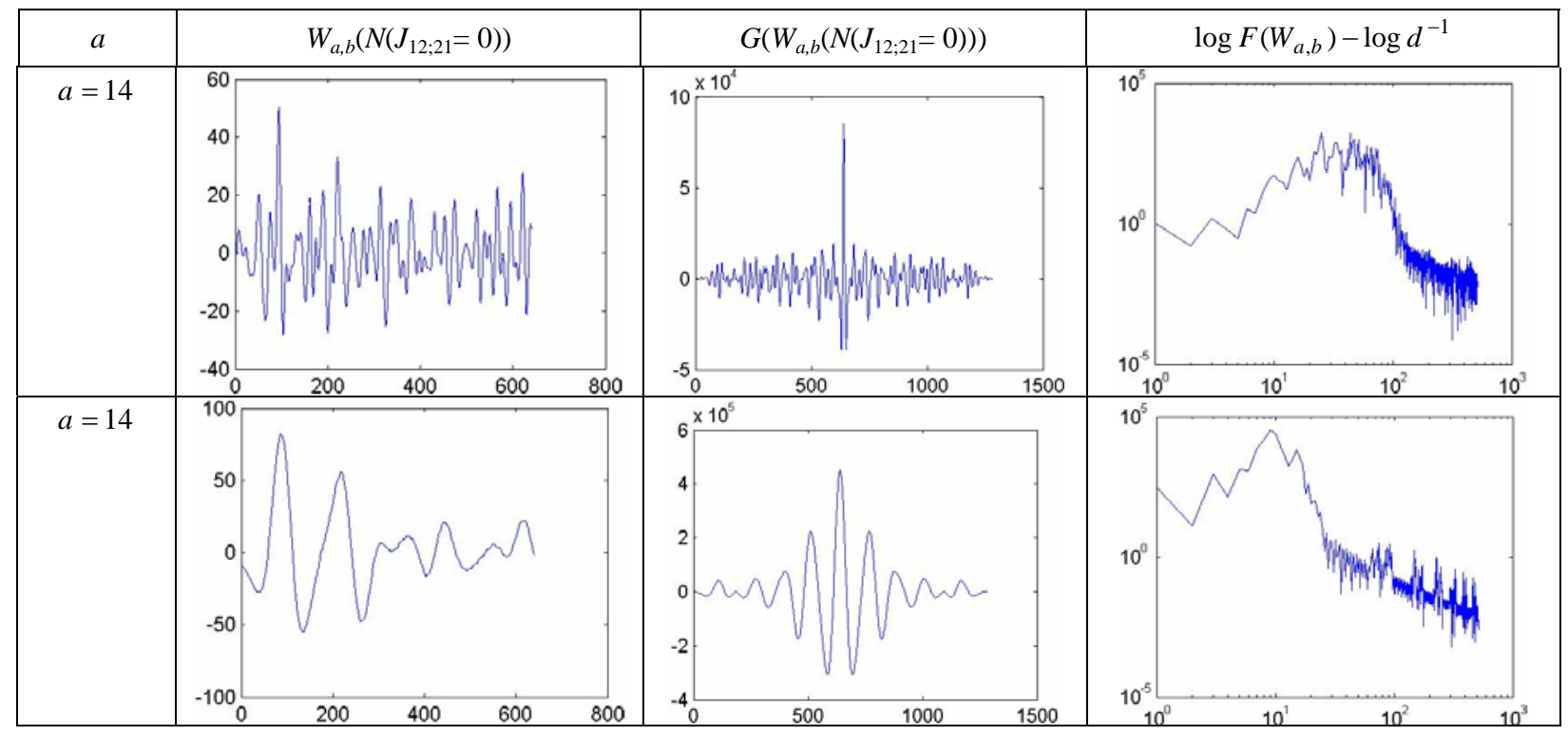

Fig. 6. Coordinate (left column), autocorrelation (central column) and spectral (right column) dependences that characterize the spectrum of wavelet coefficients $W_{a, b}\left(N\left(J_{12 ; 21}=0\right)\right.$ ) for the distribution of characteristic values in the Jones-matrix image $J_{12 ; 21}(m \times n)$ of liquid-crystal network in the layer of saliva taken from a patient sick with tuberculosis. See explanations in the text.

- $\quad$ excess $E\left(W_{a=14}\left(N\left(J_{12 ; 21}=0\right)\right)\right)$ increases by 3.85 times.

For the set of correlation moments of the 1-st to 4th orders $K_{i=1 ; 2 ; 3 ; 4}$ that characterize the autocorrelation dependences $G\left(W_{a=14}\left(N\left(J_{12 ; 21}=0\right)\right)\right)$ of distributions for wavelet coefficients $W_{a, b}\left(N\left(J_{12 ; 21}=0\right)\right)$ on the scale $a=14$ of the MHAT-function for the samples of saliva taken from the patients sick with tuberculosis, we have found the following tendencies of changes in values:

- correlation moment of the 1-st order $K_{1}$ does not practically change;

- $\quad$ correlation moment of the 2-nd order $K_{2}$ increases by 2.3 times;

- correlation moment of the 3-rd order $K_{3}$ increases by 1.8 times; 
Table 1. Statistical $(M ; \sigma ; A ; E)$, correlation $\left(K_{i=1 ; 2 ; 3 ; 4}\right)$, spectral $\left(S_{i=1 ; 2 ; 3 ; 4}\right)$ moments of the 1-st to 4-th orders for variousscale (a) distributions of wavelet coefficients $W_{a, b}\left(N\left(J_{12 ; 21}=0\right)\right.$ ) for the amount of characteristic values in Jones-matrix images corresponding to liquid-crystal network in saliva of healthy $(q=18)$ and sick with tuberculosis $(q=17)$ patients.

\begin{tabular}{|c|c|c|c|c|}
\hline State & \multicolumn{2}{|c|}{ Norm } & $a=14$ & $a=70$ \\
\hline$a$ & $a=14$ & $a=70$ & $0.12 \pm 0.034$ & $0.37 \pm 0.073$ \\
\hline$M$ & $0.086 \pm 0.018$ & $0.26 \pm 0.051$ & $0.11 \pm 0.026$ & $0.11 \pm 0.017$ \\
\hline$\sigma$ & $0.23 \pm 0.057$ & $0.085 \pm 0.012$ & $0.77 \pm 0.14$ & $0.21 \pm 0.047$ \\
\hline$A$ & $0.37 \pm 0.075$ & $0.13 \pm 0.029$ & $0.74 \pm 0.17$ & $0.24 \pm 0.053$ \\
\hline$E$ & $0.29 \pm 0.057$ & $0.19 \pm 0.035$ & $1.23 \pm 0.24$ & $0.91 \pm 0.22$ \\
\hline$K_{1}$ & $1.34 \pm 0.29$ & $0.84 \pm 0.19$ & $0.17 \pm 0.032$ & $0.13 \pm 0.027$ \\
\hline$K_{2}$ & $0.09 \pm 0.02$ & $0.16 \pm 0.035$ & $1.14 \pm 0.25$ & $0.65 \pm 0.21$ \\
\hline$K_{3}$ & $0.84 \pm 0.17$ & $0.58 \pm 0.12$ & $1.41 \pm 0.29$ & $0.87 \pm 0.19$ \\
\hline$K_{4}$ & $1.18 \pm 0.23$ & $0.76 \pm 0.15$ & $0.49 \pm 0.13$ & $0.31 \pm 0.065$ \\
\hline$S_{1}$ & $0.52 \pm 0.14$ & $0.34 \pm 0.072$ & $0.31 \pm 0.075$ & $0.15 \pm 0.041$ \\
\hline$S_{2}$ & $0.12 \pm 0.025$ & $0.11 \pm 0.024$ & $0.19 \pm 0.036$ & $0.31 \pm 0.073$ \\
\hline$S_{3}$ & $0.41 \pm 0.086$ & $0.34 \pm 0.077$ & $0.26 \pm 0.061$ & $0.29 \pm 0.063$ \\
\hline$S_{4}$ & $0.54 \pm 0.12$ & $0.34 \pm 0.085$ & & \\
\hline
\end{tabular}

- $\quad$ correlation moment of the 4-th order $K_{4}$ increases by 1.75 times.

For the set of spectral moments of the 1-st to 4th orders $S_{i=1 ; 2 ; 3 ; 4}$ that characterize distributions of extremes in the logarithmic dependences $\log J\left(W_{a, b}\right)-\log d^{-1}$ of the power spectra for wavelet coefficients $W_{a, b}\left(N\left(J_{12 ; 21}=0\right)\right)$ of the amount of characteristic values in the Jones-matrix image $J_{12 ; 21}(m \times n)$ corresponding to liquid-crystal network in saliva of patients sick with tuberculosis, we have found the following features:

- $\quad$ spectral moment of the 1-st order $S_{1}$ does not practically change;

- $\quad$ spectral moment of the 2-nd order $S_{2}$ increases by 2.1 times;

- $\quad$ spectral moment of the 3-rd order $S_{3}$ decreases by 2.2 times;

- $\quad$ spectral moment of the 4-th order $S_{4}$ decreases by 1.9 times.

The experimentally found differences in statistical, correlation and spectral moments of the 1-st to 4-th orders in $W_{a, b}\left(N\left(J_{12 ; 21}=0\right)\right)$ distributions on the certain scale $(a=14)$ of the soliton-like MHAT wavelet function can be explained by formation of additional small-scale globulin crystals with an increased level of birefringence.

\section{Conclusions}

Thus, we have demonstrated the diagnostic efficiency of the wavelet analysis applied to distributions of the amount of characteristic values in the Jones-matrix images $J_{i k}(m \times n)$ corresponding to liquid-crystalline networks of human saliva. Besides, we have offered the following parameters to diagnose tuberculosis:

- $\quad$ statistical moments of the 1-st to 4-th orders that characterize spectra of wavelet coefficients $W_{a, b}\left(N\left(J_{12 ; 21}=0\right)\right)$ for distributions of the amount $N_{12 ; 21}(x)$ of characteristic values $J_{12 ; 21}=0$ in the Jones-matrix image $J_{12 ; 21}(m \times n)$;

- $\quad$ correlation moments of the 2-nd to 4-th orders that characterize autocorrelation functions $G_{12 ; 21}(\Delta x)$ for the distributions $W_{a, b}\left(N\left(J_{12 ; 21}=0\right)\right)$;

- $\quad$ spectral moments of the 2-nd to 4-th orders that characterize distributions of extremes in logarithmic dependences $\log \left[F\left(W_{a, b}\right)\right]-\log \left(d^{-1}\right)$ for power spectra of the sets of the wavelet coefficients $W_{a, b}\left(N\left(J_{12 ; 21}=0\right)\right)$.

\section{References}

1. W.-F. Cheong, S. A. Prahl, A. J. Welch, "A Review of the Optical Properties of Biological Tissues," 
IEEE J. Quantum Electron, Vol. 26, pp. 21662185, Dec. 1990.

2. S. A. Prahl, M. Keijzer, S. L. Jacques, A. J. Welch, "A Monte Carlo model of light propagation in tissue," SPIE Proceedings of Dosimetry of Laser Radiation in Medicine and Biology, Vol. IS 5, pp. 102-111, 1989.

3. E. Wolf, "Unified theory of coherence and polarization of random electromagnetic beams ," Phys. Lett. A., Vol. 312, pp. 263-267, 2003.

4. Alexander G. Ushenko and Vasilii P. Pishak, "Laser Polarimetry of Biological Tissue: Principles and Applications", in Handbook of CoherentDomain Optical Methods: Biomedical Diagnostics, Environmental and Material Science, vol. I, Valery V. Tuchin, Ed. Boston: Kluwer Academic Publishers, 2004, pp. 93-138.

5. O. V. Angelsky, A. G. Ushenko, Yu. A. Ushenko, V. P. Pishak, "Statistical and Fractal Structure of Biological Tissue Mueller Matrix Images", in Optical Correlation Techniques and Applications, Oleg V. Angelsky, Ed. Washington: Society of Photo-Optical Instrumentation Engineers , 2007, pp. 213-266.

6. O.V. Angelsky, A.G. Ushenko, Yu.A. Ushenko, V.P. Pishak, and A.P. Peresunko, "Statistical, Correlation, and Topological Approaches in Diagnostics of the Structure and Physiological State of Birefringent Biological Tissues”, in Handbook of Photonics for Biomedical Science, Valery V. Tuchin, Ed. USA: CRC Press, 2010, pp. 21-67.

7. Alexander G. Ushenko, "Polarization structure of laser scattering fields,” Optical Engineering, vol. 34(4), pp. 1088-1093, November 1995.

8. A.G. Ushenko, "Laser diagnostics of biofractals," Quantum Electronics, vol. 29(12), pp. 1078-1084, December 1999.

9. O.V. Angel'skii, A.G. Ushenko, A.D. Arkhelyuk, S.B. Ermolenko, D.N. Burkovets, "Structure of matrices for the transformation of laser radiation by biofractals,” Quantum Electronics, vol. 29(12), pp. 1074-1077, December 1999.

10. O.V. Angel'skii, A.G. Ushenko A.D. Arheluk, S.B. Ermolenko, D. N. Burkovets, "Scattering of Laser Radiation by Multifractal Biological Structures," Optics and Spectroscopy, vol. 88(3), pp. 444-448, March 2000.

11. A.G. Ushenko, "Polarization Structure of Biospeckles and the Depolarization of Laser Radiation,” Optics and Spectroscopy, vol. 89(4), pp. 597-601, October 2000.

12. A.G. Ushenko, "Stokes-correlometry of biotissues,” Laser Physics, vol. 10(5), pp.12861292, May 2000.

13. A.G. Ushenko, "The Vector Structure of Laser Biospeckle Fields and Polarization Diagnostics of
Collagen Skin Structures," Laser Physics, vol. 10(5), pp. 1143-1149, May 2000.

14. A.G. Ushenko, "Laser polarimetry of polarizationphase statistical moments of the object field of optically anisotropic scattering layers,” Optics and Spectroscopy, vol. 91(2), pp. 313-316, February 2001.

15. A.G. Ushenko, "Polarization contrast enhancement of images of biological tissues under the conditions of multiple scattering," Optics and Spectroscopy, vol. 91(6), pp. 937-940, August 2001.

16. A.G. Ushenko, "Laser probing of biological tissues and the polarization selection of their images," Optics and Spectroscopy, vol. 91(6), pp.932-936, August 2001.

17. A.G. Ushenko, “Correlation processing and wavelet analysis of polarization images of biological tissues,” Optics and Spectroscopy, vol. 91(5), pp.773-778, June 2002.

18. A.G. Ushenko, "Polarization correlometry of angular structure in the microrelief pattern or rough surfaces," Optics and spectroscopy, vol. 92(2), pp. 227-229, June 2002.

19. O.V. Angelsky, A.G. Ushenko, Ye.G. Ushenko, "2D Stokes Polarimetry of Biospeckle Tissues Images in Pre-Clinic Diagnostics of Their Pre-Cancer States,” Journal of Holography and Speckle, vol. 2(1), pp. 26-33, April 2005.

20. Oleg V. Angelsky, Alexander G. Ushenko, and Yevheniya G. Ushenko, "Complex degree of mutual polarization of biological tissue coherent images for the diagnostics of their physiological state,” J. Biomed. Opt., vol. 10(6), Article ID 060502, November 2005.

21. O.V. Angelsky, A.G. Ushenko, and Ye.G. Ushenko, "Investigation of the correlation structure of biological tissue polarization images during the diagnostics of their oncological changes," Physics in Medicine and Biology, vol. 50(20), pp. 4811-4822, September 2005.

22. Oleg V. Angelsky, Alexander G. Ushenko, Yevheniya G. Ushenko, Yuriy Y. Tomka, "Polarization singularities of biological tissues images,” J. Biomed. Opt., vol. 11(5), Article ID 054030, September-October 2006.

23. O.G. Ushenko, S.G. Guminetsky, A.V. Motrich, "Optical properties of urine, blood plasma and pulmonary condensate of the patients with pulmovnary form of tuberculosis," Photoelectronics, vol.16, pp. 133-139, June 2007.

24. S.H. Guminetskiy, O.G. Ushenko, I.P. Polyanskiy, A.V. Motrych, F.V. Grynchuk, "The optical method for investigation of the peritonitis progressing process,” Proceedings of the SPIE, vol. 7008, Article ID 700827, April 2008.

25. Alexander Ushenko, Sergej Yermolenko, Alexander Prydij, Stepan Guminetsky, Ion Gruia, 
Ovidiu Toma, Konstantin Vladychenko, "Statistical and fractal approaches in laser polarimetry diagnostics of the cancer prostate tissues," Proceedings of the SPIE, vol. 7008, Article ID 70082C, April 2008.

26. A.G. Ushenko, A.I. Fediv, Yu.F. Marchuk, "Correlation and fractal structure of Jones matrices of human bile secret," Proceedings of the SPIE, vol. 7368, Article ID 73681Q, July 2009.

27. A.G. Ushenko, Yu.Ya. Tomka, V.I. Istratiy, "Polarization selection of two-dimensional phaseinhomogeneous birefringence images of biotissues," Proceedings of the SPIE, vol. 7388, Article ID 73881L, December 2009.

28. A.G. Ushenko, A.I. Fediv, Yu.F. Marchuk, "Singular structure of polarization images of bile secret in diagnostics of human physiological state," Proceedings of the SPIE, vol. 7368, Article ID 73681S, July 2009.
29. S.B. Yermolenko, A.G. Ushenko, P. Ivashko, "Spectropolarimetry of cancer change of biotissues," Proceedings of the SPIE, vol. 7388, Article ID 73881D, December 2009.

30. A.G. Ushenko, I. Z.Misevich, V. Istratiy, I. Bachyns'ka, A. P. Peresunko, Omar Kamal Numan, and T. G. Moiysuk, "Evolution of Statistic Moments of 2D-Distributions of Biological Liquid Crystal Net Mueller Matrix Elements in the Process of Their Birefringent Structure Changes,” Advances in Optical Technologies, vol. 2010, Article ID 423145, March 2010.

31. O.V. Dubolazov, A.G. Ushenko, V.T. Bachynsky, A.P. Peresunko, and O.Ya. Vanchulyak, "On the Feasibilities of Using the Wavelet Analysis of Mueller Matrix Images of Biological Crystals," Advances in Optical Technologies, vol. 2010, Article ID 162832, March 2010. 